\title{
Research of Hyperbaric Oxygen Pretreatment to Improve The Lower Body Negative Pressure Endurance of Pilots with Poor Acceleration Endurance
}

\author{
Mengyu Ma \\ Hebei North University $\llbracket$ Zhangjiakou HeBei \\ Hongjin Liu ( $13601157961 @ 139 . c o m$ ) \\ Air Force General Hospital PLA \\ Zhaojun Fu \\ Air Force General Hospital PLA \\ Qiming Liu \\ Air Force General Hospital PLA \\ Rongli Sun \\ Air Force General Hospital PLA
}

\section{Research Article}

Keywords: Acceleration, The pilot, Hyperbaric oxygen, Lower body negative pressure

Posted Date: January 24th, 2022

DOI: https://doi.org/10.21203/rs.3.rs-1256255/v1

License: (c) This work is licensed under a Creative Commons Attribution 4.0 International License. Read Full License 


\section{Abstract}

Background: To investigate the effect of hyperbaric oxygen preconditioning (HBOP) on improving the lower body negative pressure endurance of pilots with poor acceleration endurance.

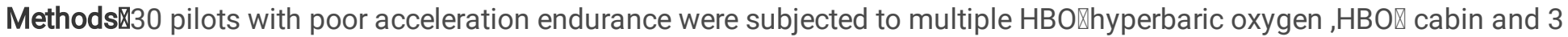
times HBO exposure. Oxygen inhalation was performed for $95 \mathrm{~min}$, once a day (time was fixed at 14:30-16:15 PM in the HBO cabin), for consecutive $3 \mathrm{~d}$. The lower body negative pressure endurance and blood indexes were measured before and after HBOP using the subjects' own before-after control design. Lower body negative pressure endurance adopts stepwise increasing scheme, that is, continuously increasing LBNP load:-20mmHg· $1 \mathrm{~min} \rrbracket-30 \mathrm{mmHg} \cdot 3 \mathrm{~min} \rrbracket-40 \mathrm{mmHg} \cdot 5 \mathrm{~min} \rrbracket-50 \mathrm{mmHg} \cdot 7 \mathrm{~min}$, etc .

Results:The cumulative stress index of subjects before HBOP was $(-856.13 \pm 273.76 \mathrm{~min} \cdot \mathrm{mmHg})$, and that after HBOP was $(-1237.42 \pm 385.50 \mathrm{~min} \cdot \mathrm{mmHg})$. The cumulative stress index after HBOP increased significantly $(t=7.750, P<0.05)$, namely lower body negative pressure endurance significantly improved. After that, 30 people underwent centrifuge reexamination, and the passing rate of 4.0G10s reached 93.3\%.After HBOP, diastolic blood pressure increased $(t=-3.252, P<0.05)$, heart rate slowed down ( $t=5.493, P<0.05)$, blood oxygen saturation $\left(\mathrm{SaO}_{2}\right)$ increased $(t=-3.08, P<0.05)$, but there was no significant difference in systolic blood pressure.Blood lactic acid (BLA) was lower than that before HBOP ( $t=2.186$, $\mathrm{P}<0.05)$; Adrenocorticotropic hormone $(\mathrm{ACTH})$ was not significantly different from that before HBOP.

Conclusion:HBOP can improve the lower body negative pressure endurance and centrifuge acceleration endurance of pilots with poor acceleration endurance, and its mechanism may be related to the improvement of autonomic nerve function and the enhancement of human energy metabolism.

\section{Background}

With the rapid replacement of our fighter jets and the continuous change of flight mode and combat style, the acceleration load continues to increase. The acceleration changes sharply in flight, up to the persistence of $9 \mathrm{G}$ or even higher $\mathrm{G}$ value +

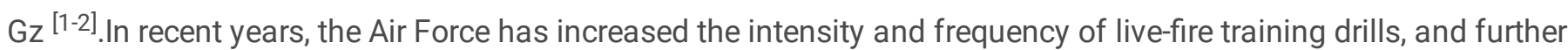
improved the technical standards of pilot training. The acceleration of gray vision, black vision and even G-LOC (G-induced loss of consciousness, G-LOC) occur from time to time, which are important factors threatening the flight safety of the Air Force.Therefore, how to more effectively improve the acceleration endurance of pilots is the current hot topic of military aviation medicine discussion ${ }^{[3]}$.

At present, the centrifuge is not only the gold standard equipment to test the pilot acceleration endurance, but also has been recognized by the majority of scholars as the acceleration endurance training equipment.But the centrifuge equipment is huge, expensive, and difficult to promote. It has been shown that the biological effect of ${ }^{[4]}$ LBNP is similar to the centrifuge $+\mathrm{G}_{Z}$, that is, blood redistribution during LBNP, causing decreased blood pressure and insufficient blood supply to the head.. Negative body pressure at sitting $-6.67 \mathrm{kPa}$ can simulate the effect of $+(2 \sim 3) \mathrm{G}_{Z}$ on human cardiovascular function. Therefore, the lower body negative pressure endurance can reflect the acceleration endurance.The lower body negative pressure chamber equipment is small and easy to operate, which can be used for the preliminary detection of the acceleration endurance, but also for the acceleration endurance training ${ }^{[5]}$. In this experiment, the lower body negative pressure endurance was tested by the lower body negative pressure chamber and the acceleration endurance was tested by centrifuge.

In China, ${ }^{[6-7]}$ hyperbaric oxygen pretreatment (hyperbaric oxygen preconditioning, HBOP) to improve autonomic nerve function, improve energy metabolism, affect the HPA axis during stress to improve the pilot standing endurance, standing endurance is closely related to acceleration endurance. In foreign studies ${ }^{[8-9]}$, HBOP can improve autonomic nerve function, 
increase blood pressure significantly, heart rate significantly decreased, and improve the body's energy metabolism. Liu Hongjin ${ }^{[10]}$ team has published the article "Application Research of hyperbaric Oxygen Pretreatment to improve the negative pressure endurance of pilots", which confirmed that HBOP can effectively improve the negative pressure endurance of normal pilots. This study investigated whether HBOP can improve the lower body negative pressure endurance (Lower body negative pressure, LBNP) and acceleration endurance, and initially explored the mechanism.

\section{Materials And Methods}

\section{Study subjects}

A total of 30 male fighter pilots with poor acceleration endurance ( $\mathrm{Gz}$ endurance $<4.0 \mathrm{G} / 10 \mathrm{~s}$ ) were selected from February 2021 to July 2021 in the Air Force Special Medical Center , age (26.20 \pm 4.20$)$, height $(174.39 \pm 2.40) \mathrm{cm}$, weight $(67.84 \pm 5.35) \mathrm{kg}$, flight time $(650.19 \pm 510.77) \mathrm{h}$, no various basic diseases and genetic history, and daily physical training.Physical and mental health was proved by blood drawing testing, plate exercise test, heart ultrasound, abdominal ultrasound, chest, whole spine X-ray, and psychological evaluation.The experiment was approved by the hospital ethics committee, and clearly informed all subjects of the specific research content, precautions and possible technical risks, and signed an informed consent form for the experiment.

\section{Study Methods}

The subjects measured the negative pressure endurance, centrifugal acceleration endurance and related blood indicators before and after HBOP.

HBOP scheme: using the multiplayer oxygen cabin, 3 times of oxygen inhalation HBOP scheme.After the subject enters the closed cabin door, the air is gradually pressurized to 2.5ATA for $10 \mathrm{~min}$, stabilized for $65 \mathrm{~min}$ (30min 2, stop oxygen for $5 \mathrm{~min}$ ), and gradually to $1.3 \mathrm{ATA}$ within $10 \mathrm{~min}$ and stay for $5 \mathrm{~min}$ continue decompression for $5 \mathrm{~min}$ and end exit..A total of 95min, once daily (fixed at 14:30-16:15 p. m.) for 3d continuously.

Test method of lower body negative pressure endurance: adopt the step increase scheme, that is, to continuously increase the LBNP load, and increase-20mmHg $1 \mathrm{~min},-30 \mathrm{mmHg} 3 \mathrm{~min},-40 \mathrm{mmHg} 5 \mathrm{~min},-50 \mathrm{mmHg} 7 \mathrm{~min}$, etc.During the test, ask and closely observe the subject's subjective feelings and signs. When the endurance end point is reached, the negative pressure in the lower body negative press meter should be quickly removed to end the experiment.Endurance endpoint index (either of the following): blood pressure: sudden drop to below 90 / 60mmHg; heart rate: heart rate suddenly dropped 15 times, or below 60 times per minute; or the subjects complained of dizziness, palpitation, chest tightness and nausea, and symptoms such as pale face and cyanosis.

Centrifuge detection method (before and after HBOP test): $+: 2 \mathrm{Gz},+3 \mathrm{Gz}$ and $+4 \mathrm{Gz}$ test at 8:30 to 11:00 am, with $\mathrm{G}$ growth rate of $3 \mathrm{G} / \mathrm{s}$ and load duration of $10 \mathrm{~s}$. The judgment index of the pilot peripheral visual loss (peripheral light loss, PLL) or the decrease in the amplitude of the ear pulse wave by more than $75 \%$ or that the pilot was unable to continue the experiment for various reasons.

Examination method of blood indicators: a total of 2 blood samples. The time points of the experiment were: A: On the first day of the experiment, $2 \mathrm{ml}$ of elbow venous blood was extracted from each subject before oxygen inhalation (fixed at 8:00 $\mathrm{am}$ ), and the blood was smoothly sent to the Inspection Department of Air Force Specail Medical Center by centrifugation to complete the detection of $\mathrm{SaO}_{2}, \mathrm{BLA}$ and ACTH content before HBOP; $\mathrm{B}$ : After the last one oxygen inhalation, the elbow venous blood was extracted again at 8:00 the next day.

\section{Statistical analysis}


Data analysis was SPSS25.0 statistical software. All experimental quantitative data are expressed as mean \pm standard deviation $(x \pm s)$. The experimental data met the normal distribution.Meometric data tested for normality were compared using a paired t-test and considered statistically significant at $\mathrm{P}<0.05$.

\section{Results}

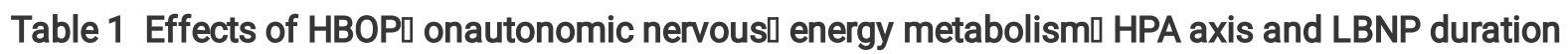

\begin{tabular}{|c|c|c|c|c|c|c|c|}
\hline & LBNP $\triangle \min \rrbracket$ & $\begin{array}{l}\text { Systolic } \\
\text { pressure }(\mathrm{mmHg})\end{array}$ & $\begin{array}{l}\text { Diastolic } \\
\text { pressure(mm } \\
\mathrm{Hg})\end{array}$ & $\begin{array}{l}\text { Heart rate } \\
\text { खbeat/min } \rrbracket\end{array}$ & $\begin{array}{l}\text { Blood oxyen } \\
\text { sat } \\
\text { uration(\%) }\end{array}$ & $\begin{array}{l}\text { BLA } \\
\text { \mmol/LV }\end{array}$ & $\begin{array}{l}\text { ACTH } \\
\text { 『pmol/L】 }\end{array}$ \\
\hline $\begin{array}{l}\text { Pre- } \\
\text { HBOP }\end{array}$ & $15.37 \pm 3.73$ & $110.84 \pm 8.77$ & $71.06 \pm 6.11$ & $64.97 \pm 8.68$ & $74.91 \pm 17.87$ & $2.66 \pm 1.38$ & $48.31 \pm 21.30$ \\
\hline $\begin{array}{l}\text { Pro- } \\
\text { HBOP }\end{array}$ & $20.48 \pm 4.61$ & $109.19 \pm 6.94$ & $75.55 \pm 7.09$ & $56.74 \pm 7.61$ & $82.45 \pm 16.76$ & $2.13 \pm 0.70$ & $45.87 \pm 14.36$ \\
\hline $\begin{array}{l}\mathrm{t} \\
\text { value }\end{array}$ & 7.750 & 0.930 & -3.252 & 5.493 & -3.08 & 2.186 & 0.654 \\
\hline $\begin{array}{l}P \\
\text { value }\end{array}$ & 0.001 & 0.36 & 0.003 & 0.032 & 0.005 & 0.037 & 0.518 \\
\hline
\end{tabular}

${ }^{*} \mathrm{P}<0.05$ as compared with Con

\section{Comparison of the CSI before and after HBOP}

After 3 HBOP, the lower body negative pressure endurance increased significantly.CSI: cumulative stress index = negative pressure level $(\mathrm{mmHg}) \times$ each negative pressure level tolerance time $(\mathrm{min})$. The cumulative stress index was

$(-856.13 \pm 273.76) \mathrm{min} \cdot \mathrm{mmHg}$ before oxygen inhalation, and $(-1237.42 \pm 385.50) \mathrm{min} \cdot \mathrm{mmHg}$ after oxygen inhalation, which showed that the subjects increased significantly and was statistically significant $(t=7.750, P=0.001)$.(See Table 1)

\section{Changes in acceleration endurance before and after HBOP}

After the experiment, the centrifuge test was conducted. 28 of the 30 fighter pilots with poor acceleration endurance passed the centrifuge test (4.0G / 10s), and one of them did not participate in the centrifuge test due to achilles tendon injury.

\section{Comparison of autonomic indicators before and after HBOP}

After 3 HBOP, the subjects' diastolic BP increased significantly $(t=-3.252, P=0.003)$, heart rate slowed significantly ( $t=5.493$, $P=0.032)$, oxygen saturation (SaO2)increased significantly $(t=-3.08, P=0.005)$; no significant difference before systolic $B P$ HBOP.(See Table 1)

\section{Comparison of energy metabolism before and afterHBOP}

The BLA before HBOP was $2.66 \pm 1.38 \mathrm{mmol} / L$, and the BLA after HBOP was $2.13 \pm 0.70 \mathrm{mmol} / \mathrm{L}, t=2.186, P=0.037$, the difference was statistically significant. (See Table 1)

\section{Comparison of ACTH before and after HBOP}


The ACTH was $48.31 \pm 21.30 \mathrm{mmol} / \mathrm{L}$ before HBOP, and $45.87 \pm 14.36 \mathrm{mmol} / \mathrm{L}$ after HBOP. The difference was not statistically significant $(T=0.654, P=0.518)$. (See Table 1$)$

\section{Discussion}

\section{HBOP can improve the lower negative pressure endurance and acceleration endurance of pilots}

The results showed a significant increase in the cumulative stress index after HBOP among the 30 pilots.A centrifuge review was performed, and the $4.0 \mathrm{G} 10 \mathrm{~S}$ pass rate reached $93.3 \%$. The pilot who failed to pass the centrifuge test had a history of overheating shooting ten years ago. Although they are currently healthy, the acceleration is still not reaching the standard, and they will follow up with the training of the pilot's acceleration endurance.

Head foot to positive acceleration endurance namely $+G z$ endurance is one of the main factors affecting flight safety, this paper acceleration endurance refers to $+\mathrm{Gz}$ endurance ${ }^{[11]}$.

Liu Hongjin team ${ }^{[10]}$ in the "hyperbaric oxygen pretreatment to improve the pilot lower body negative pressure endurance application research" has confirmed that HBOP can improve the lower body negative pressure endurance of normal fighter pilots.As can be seen in this experiment, after HBOP, the lower body negative pressure endurance of the poor acceleration endurance pilots was significantly improved and verified by the centrifuge test. $93.3 \%$ of the poor acceleration endurance (+ Gz endurance <4.0G / 10s) pilots had reached the standard in the centrifuge test.The mechanism that HBOP can improve the negative pressure endurance and acceleration endurance is discussed from the following aspects.

\section{Preliminary discussion on the mechanism of negative pressure endurance by HBOP}

\section{HBOP improves autonomic nervous function}

This experiment found that HBOP improved the diastolic blood pressure of the pilots with poor acceleration endurance, slowed the heart rate, increased the blood oxygen saturation, made the pilot's autonomic nervous system more stable, and the sympathetic and parasympathetic nerves can better coordinate with each other, thus improving the lower negative pressure endurance ${ }^{[12]}$.

Studies have been shown that hyperbaric oxygen (HBO) can increase arterial pressure and reduce heart rate ${ }^{[13]}$.Increasing arterial pressure is due to: 1) the direct effect of HBO on peripheral arterial vessels, stimulating the synthesis and release of active chemicals such as endothelin-1 and thromboane in the body, causing rapid contraction of peripheral blood vessels, vascular resistance to greatly increase ${ }^{[14]}$; 2) $\mathrm{HBO}$ can reduce $\mathrm{CO}_{2}$ content in blood, thus reducing its dilating effect on [13]; 3) $\mathrm{HBO}$ also has a-adrenaline-like effect, which can directly make vaso constriction and increase hypertensive ${ }^{[15]}$. It has been believed that ${ }^{[16-17]}$, healthy people increase total vascular resistance by more than $50 \%$ under 3ATA oxygen pressure, can lead to increased diastolic blood pressure. The main factors affecting the diastolic blood pressure are the heart rate and the peripheral resistance, while the main factors affecting the systolic blood pressure are the cardiac output per beat and the elasticity of the arterial wall.Under HBO, the slowing heart rate decreases the cardiac output, the systolic blood pressure decreases while the diastolic blood pressure increases, and the pulse pressure difference decreases by ${ }^{[18]}$.

HBO treatment can slow down heart rate probably because the chemoreceptors and pressure receptors of the carotid sinus and aortic arch are stimulated by high concentrations of blood oxygen and increased intracascular pressure, which reflex slows heart rate, which further causes insufficient blood oxygen supply ${ }^{[19]}$ in all organs, especially the human brain; At the same time, during HBO treatment, the obvious decrease in nitric oxide concentration makes it directly act in sinus node disciplined cells, and also reduces heart rate ${ }^{[20]}$.It has been shown that HBO exposure can increase the high-frequency 
power in the frequency domain index of heart rate variability, thus increasing the vagal nerve activity, slowing down the heart rate, reducing its load on the heart wall, and reducing the myocardial oxygen consumption by [21].

The amount of oxygen fed into the tissue by HBO treatment is increased, so the oxygen saturation levels in posterity after HBO exposure significantly increase ${ }^{[22]}$.

HBOP can promote the heart to show signs of "functional saving", which can prolong the duration of physical load, enhance the body movement ability and thus improve the lower body negative pressure endurance [23].

\section{HBOP enhances the human energy metabolism}

In this experiment, it was found that BLA decreased significantly after HBOP compared with before, indicating that HBOP may be involved in the metabolic clearance of lactic acid in the body, which is beneficial to prolong the time of physical load and further improve the acceleration endurance.

Fatigue refers to the energy needed to fully release muscle strength and muscle contraction, due to the accumulation of lactic acid, hydrogen ions, inorganic phosphate and ammonia and other metabolites and inhibit muscle contraction, so, the accumulation of lactic acid will lead to fatigue, and the rapid elimination of fatigue will help to improve the exercise ability ${ }^{[24]}$.According to the domestic military ${ }^{[25]}$, at an altitude of $3700 \mathrm{~m}$, five HBOP can effectively improve the oxygen radical metabolism of up to $8 \mathrm{~d}$ and two HBOP of up to $5 \mathrm{~d}$.As HBOP can accelerate the clearance rate of BLA in the body at high altitude, the energy supply of the body is transformed from anaerobic glycolysis of sugar to aerobic oxidation of sugar, which not only rapidly reduces the production of BLA, but also facilitates the clearance of BLA and other acidic products due to the significant increase of oxygen tension in tissues, cells and body fluids [9].At the same time, short-term HBOP can significantly and effectively improve the blood glucose level after physical load, significantly reduce the activity of lactate dehydrogenase and $\mathrm{Na}^{+}-\mathrm{K}^{+}$-ATP enzyme, and accelerate the clearance of BLA invivo, which is very important to effectively reduce the occurrence of fatigue, reduce the fatigue severity and effectively improve the labor efficiency of [26].

HBOP used in pilots with poor acceleration endurance can increase the blood oxygen content of the body, further expand the effective area when the blood flows through each tissue, and then improve the cardiovascular function during the physical load, reduce the lactate concentration, reduce the fatigue degree, and improve the acceleration endurance ${ }^{\text {[27] }}$.

\section{HBOP affects the hypothalamic-pituitary-adrenocortical axis (HPA axis)?}

Due to the detection of ACTH blood indicators involved in the experiment, there was a circadian rhythm of normal ACTH secretion, high in the morning and low in the afternoon and evening, and the blood draw time was uniformly set at 8:00 am to control the experimental variables.

After result analysis, the ACTH change after HBOP in this experiment was not statistically significant.In this study, the pilots were in a quiet state before and after HBOP, and showed no acute stress response, and failed to monitor the changes of HBOP in ACTH under stress. The HPA axis is the neuroendocrine system with sensitive to stress response in the body. In the environment of acute stress, people experience a corresponding response and gradually adapt to the new environmental ${ }^{[28]}$.It has been shown that in ${ }^{[29]}$, reacute high-altitude exposure after HBOP can continuously increase both central neurotransmitter and ACTH.The mechanism may be that HBOP keeps the body's HPA axis continuously excited during stress, which enables the human body to produce an enhanced stress response under high altitude, low oxygen, insufficient physical load oxygen supply and HBOP to adapt to the new environment faster.HBOP can effectively regulate the secretion of the HPA axis to a series of hormones such as noradrenaline and corticotrotrophin under stress.

The factors related to HBOP for stress experiments will be studied in the future. 


\section{Conclusion}

It is proved that HBOP can improve autonomic nervous function and energy metabolism, so as to improve lower body negative pressure endurance and acceleration endurance of pilots with poor acceleration endurance. Therefore, HBOP before air combat may be a fast and effective method to improve the acceleration endurance of pilots with poor acceleration endurance.

In future experiments, HBOP combined with HBOP and negative pressure training, so as to further explore effective and convenient methods to improve the acceleration endurance of pilots.

\section{Abbreviations}

HBOP:Hyperbaric oxygen preconditioning; HBO:Hyperbaric oxygen; G-LOC:G-induced loss of consciousness; LBNP:Lower body negative pressure; PLL:Peripheral light loss; SaO2:Blood oxygen saturation ; BLA:Blood lactic acid ; ACTH:Adrenocorticotropic hormone ;CSI:Cumulative stress index;

\section{Declarations}

\section{Acknowledgements}

Heartfelt thanks to the developer of the lower body negative pressure chamber, the helpers of HBOP and the volunteers who contributed to this experiment.

\section{Author Contributions}

All authors contributed to the study.Conception and design were performed by LH.Material preparation, data collection were performed by MM/FZ/ LQ/ SR.Analysis were performed by MM. The first draft of the manuscript was written by $\mathrm{MM} / \mathrm{LH}$, and all authors commented on previous versions of the manuscript. All authors read and approved the final manuscript.

\section{Funding}

The article was funded by Air Force Logistics Research Program (KGJ10J161)

\section{Availability of data and materials}

All data generated or analyzed during this study are included in this published article.

\section{Ethics approval and consent to participate}

The experiment was approved by the Air Force General Hospital PLA ethics committee, and clearly informed all subjects of the specific research content, precautions and possible technical risks, and signed an informed consent form for the experiment.

\section{Competing interests}

The authors declare that they have no competing interests.

\section{Consent for publication}

Not applicable. 


\section{References}

1. Liang Minglong, XU Jianhua.Military Aircrew Fitness Promotion Concept and Concept [J].Chinese Journal of Health And Medicine, 2021,23(1):105-107.(in Chinese)

2. Li Xi, GENG Xichen.Study on physiological stress response and mechanism of high + Gz exposure in human body [D]. Fourth Military Medical University, 2003.(in Chinese)

3. Xu Xianrong, FU Zhaojun, Yin Xin et al.Analysis of inpatient disease spectrum of fighter pilots [J]. Chin $\mathrm{J}$ aerospace med,2005,16 (2) : 135-138.(in Chinese)

4. Yao Yongjie, Sun Yuyang, Sun Xixi et al. Effect of head tilt combined with $-9.33 \mathrm{kPa}$ lower body negative pressure on cardiovascular function after exposure to inverted position [J]. Journal of the fourth military medical university,2003,24(2):156-159.

5. Fu Zhaojun, XU Xianrong, Liu Hongjin, Zheng Jun, et al. Improvement of Acceleration Endurance of Fighter Pilots by Lower Body Negative Pressure Exercise [J]. Journal of general hospital of air force,2009,25(3):106-107. (in Chinese)

6. Sun Xi qing, Shi Fei, Gao Yuan, et al. Hyperbaric oxygen pretreatment can improve human orthostatic endurance [C]// Hyperbaric Oxygen Medicine Branch of Chinese Medical Association. Chinese Medical Association, The 24th National Compilation of Academic Papers on Hyperbaric Oxygen Medicine, Shenyang, 2015. Beijing: Chinese Society of Hyperbaric Oxygen Medicine, 2015:89-93 (in Chinese)

7. Zhang Lifan, Zheng Jun, Wang Xingbang et al. Effect of aerobic exercise on orthostatic endurance and circulatory response [J]. Chinese journal of aerospace medicine,1997,8 (4) :13-17.

8. Ishihara A. Mild hyperbaric oxygen: mechanisms and effects. J Physiol Sci. 2019;69(4):573-580.

9. Al-Waili NS, Butler GJ, Beale J, et al. Influences of hyperbaric oxygen on blood pressure, heart rate and blood glucose levels in patients with diabetes mellitus and hypertension. Arch Med Res. 2006;37(8):991-997.

10. Liu Ye, Liu Hongjin.Application of hyperbaric oxygen pretreatment to Improve the Endurance of pilots under lower Body Negative Pressure [J].Chinese journal of aeronautics and astronautics medicine,2018,29(2):86-89.(in Chinese)

11. Xian-rong xu.Accurate Understanding and Application of clinical aviation Medical Terminology [J].Journal of PLA medical college, 2014,35(8):781-786.(in Chinese)

12. Zuo Xin, Peng Li, Yang Anqiang et al.Heart rate variability in patients with acute stress [J]. Chinese journal of behavioral medicine \& brain science,2013,22(4):345-347.(in Chinese)

13. Lund VE, Kentala E, Scheinin H, Klossner J, Helenius H, Sariola-Heinon-en K, Jalonen J. Heart rate variability in healthy volunteers during normobaric and hyperbaric hyperoxia. Acta Physiol Scand 1999,167(1): 29-35.

14. Mialon P, Barthélémy L. Effect of hyperbaric oxygen on prostaglandin and thromboxane synthesis in the cortex and the striatum of rat brain. Mol Chem Neuropathol. 1993;20(2):181-189.

15. Freiberger JJ, Derrick BJ, Natoli MJ, et al. Assessment of the interaction of hyperbaric N2, CO2, and 02 on psychomotor performance in divers.J Appl Physiol (1985). 2016;121(4):953-964.

16. Wu Xiaoyang. Principle and indication of hyperbaric oxygen therapy [J]. Clinical Rational Drug Use,2014,7(1):85.(in Chinese)

17. Li Ning, HUANG Huai.Clinical therapy of hyperbaric oxygen [M]. Beijing: China Union Medical College Press,2207:3334,142.(in Chinese)

18. LI Hongling, XUE Xinping, et al. Influence of hyperbaric oxygen treatment environment on blood pressure and pulse in cabin [J].Journal of hebei medical university,2013,34(2):167-171. (in Chinese)

19. CAI Hongbin, GE Chaoming et al.Protective effect of hyperbaric oxygen pretreatment on free radical injury induced by focal cerebral ischemia-reperfusion in rats [J].Chinese Journal of Rehabilitation Medicine,2011,26(9):803-806.(in Chinese) 
20. Wang Xia, Shi Lei, Wang Liming et al.Analysis on the characteristics and trend of heart rate change in patients with hyperbaric oxygen therapy [J]. Chin J Marine med \& hyperbaric med,2020,27(5):594-596.(in Chinese)

21. Pabla R, Curtis MJ. Effects of NO modulation on cardiac arrhythmias in the rat isolated heart. Circ Res. 1995,77(5):984-992.

22. Shi Ran, Ma Lizhu.Effect and nursing of hyperbaric oxygen therapy in ischemic stroke [J]. Yunnan medicine,2016,345(5):582-583.(in Chinese)

23. Liu Hongtao, LI Yangyang et al.Effects of hyperbaric oxygen pretreatment on maximal oxygen uptake and bench test index after acute exposure at simulated altitude of $4000 \mathrm{~m}$ [J].Chinese journal of navigation medicine and hyperbaric medicine,2015,22(3):169-172.(in Chinese)

24. Park SH, Park SJet al. The effects of low-pressure hyperbaric oxygen treatment before and after maximal exercise on lactate concentration, heart rate recovery, and antioxidant capacity.J Exerc Rehabil. 2018;14(6):980-984.

25. Cui Jianhua, GAO Liang, Zhang Xizhou et al.Chinese journal of applied physiology,2008,24(4):444-447.(in Chinese)

26. Shi Lu, LI Yangyang, ZHANG Yanmeng et al.Effect of hyperbaric oxygen pretreatment on labor energy metabolism in human body with hypoxia at high altitude [J]. Chinese journal of navigation medicine and hyperbaric medicine,2015,22(2):96-99.(in Chinese)

27. Ma Guangquan, Cui Jianhua et al.Effect of hyperbaric oxygen on lung function of young people in high altitude area [J]. Xizang medical journal,2009,30(2):1-3.(in Chinese)

28. Zhang Quan, Wang weiwen, et al.Psychological Science, 2016, 24(4):536-546.(in Chinese)

29. Li yangyang, Shi lu, Wu nanning, et al. Effect of hyperbaric oxygen pretreatment on human stress response to acute exposure to physical stress at high altitude [J]. Chinese journal of occupational diseases,2015,33(10):731-734.(in Chinese) 